\title{
Coronary Artery Thrombosis: Is High Serum Homocysteine Level a Culprit?
}

\author{
Laxman Dubey, Sogunuru Guruprasad, Rabindra Bhattacharya, Gangapatnam Subramanyam \\ Department of Cardiology \\ College of Medical Sciences and Teaching Hospital, Bharatpur-10, Nepal
}

\author{
Citation \\ Laxman Dubey, Sogunuru Guruprasad, Rabindra Bhattacharya, \\ et al. Coronary Artery Thrombosis: Is High Serum \\ Homocysteine Level a Culprit? Nepalese Heart Journal \\ 2014;11(1): 49-52.

\section{Keywords} \\ coronary artery thrombosis; acute coronary syndrome; \\ hyperhomocysteinemia; thrombolysis
}

\begin{abstract}
Hyperhomocysteinemia is a risk factor of coronary artery disease associated to arterial thrombosis. We report a case in which coronary angiography showed full of thrombus causing total obstruction of the left anterior descending artery. Patient was managed successfully with intravenous thrombolysis. Biochemical analysis revealed a high serum homocysteine level.
\end{abstract}

\section{INTRODUCTION}

Hyperhomocysteinemia is characterized by an abnormally high concentration of homocysteine (a sulfurated amino acid produced through the methionine metabolism). It is associated to arterial thrombosis and is a risk factor of coronary artery disease. Hyperhomocysteinemia is classified as mild to moderate $(15-30 \mu \mathrm{mol} / \mathrm{L})$, intermediate (>31-100 $\mu \mathrm{mol} / \mathrm{L})$, and severe $(>100 \mu \mathrm{mol} / \mathrm{L}){ }^{1}$ It has been shown that subjects with moderate hyperhomocysteinemia are characterized by a prothrombotic, and dysfibrinolytic state and homocysteine level is an independent predictor of thrombotic events. ${ }^{2}$ Moreover, increased plasma homocysteine had been shown to be associated with increased cardiovascular risk independently of other atherosclerosis risk factors. ${ }^{3}$

\section{CASE REPORT}

A 52-year-old male presented to our emergency department with left sided chest heaviness for two weeks. He was a smoker who had no history of hypertension, dyslipidemia, diabetes mellitus, or family history of coronary artery disease. He was not under any medications. His body mass index was 23 and the vital signs were normal (blood pressure: 120/80 $\mathrm{mmHg}$, heart rate: 72 beats per minute). Electrocardiography (ECG) showed normal sinus rhythm but no evidence of myocardial ischemia (Figure 1). CK-MB was $18 \mathrm{U} / \mathrm{L}$ and troponin I was negative. Transthoracic echocardiography showed grade I left ventricular (LV) diastolic dysfunction, no regional wall motion abnormality, and normal LV ejection fraction. No intracardiac mass or thrombus was noted. There was no history of drug abuse. He was treated with aspirin, statin, beta blocker and nitrates, however, he had ongoing chest pain.

\section{Corresponding author}

Dr. Laxman Dubey

Department of Cardiology

College of Medical Sciences and Teaching Hospital,

Bharatpur-10, Nepal

E-mail:dubeylax@yahoo.com 


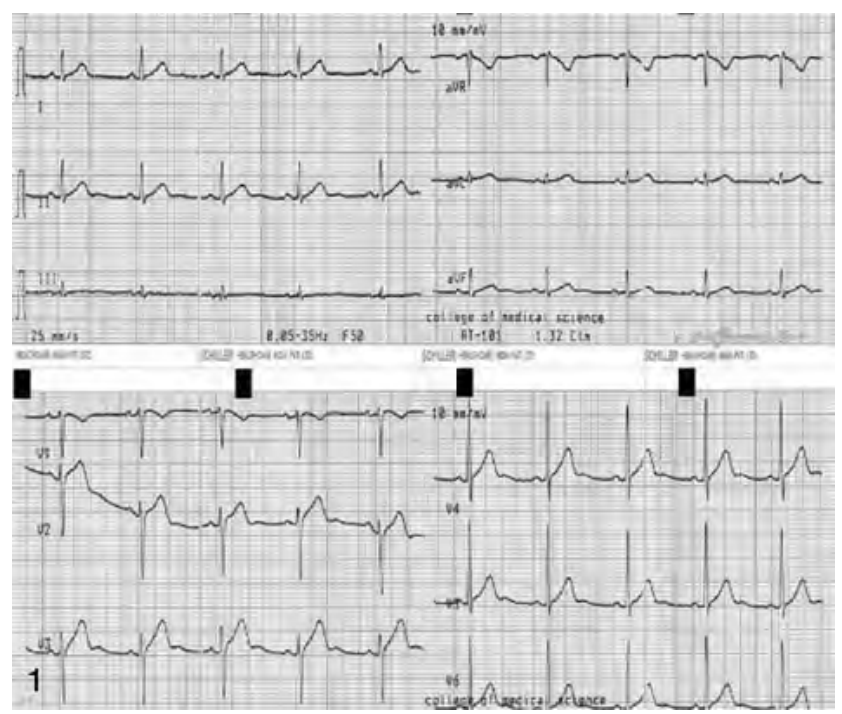

Figure 1

Figure 1. Electrocardiography on presentation showing normal sinus rhythm with no features of myocardial ischemia

He underwent coronary angiography (CAG) from right femoral approach using 6 French Judkins diagnostic catheters, which revealed full of thrombus at the proximal left anterior descending artery (LAD) as well as the first diagonal branch causing total occlusion (Figure 2a). Immediately after the first injection to the left coronary artery, patient developed severe retrosternal chest pain and difficulty breathing. ECG monitor showed extensive ST-segment elevation. Another injection in the same angiographic view revealed no dissection but full of thrombus causing complete occlusion at the ostial LAD (Figure 2b). His blood pressure decreased to $70 / 40 \mathrm{mmHg}$ and heart rate gradually started to drop and then arrested into asystole. Chest compression started. Atropine was given and he was started on inotropes. There were two possible strategies in the management of our case. One is the interventional strategy (stenting preceded by thrombus aspiration) and another is the pharmacological strategy (GP IIb/IIIa inhibitor or thrombolysis), eventually followed by an interventional strategy. However, thrombus aspiration catheter was not available at that time and we decided to thrombolyze the patient. Emergency thrombolysis with streptokinase 1.5 million units diluted in $100 \mathrm{ml} 0.9 \%$ normal saline over 45 minutes was completed. Thrombolytic period was uneventful and the patient's general condition improved. Blood pressure increased to $120 / 70 \mathrm{mmHg}$ and heart rate increased to 110 beats per minute. Further intervention was postponed and the patient was transferred to coronary care unit and put on low molecular weight heparin, aspirin, clopidogrel, and atorvastation.

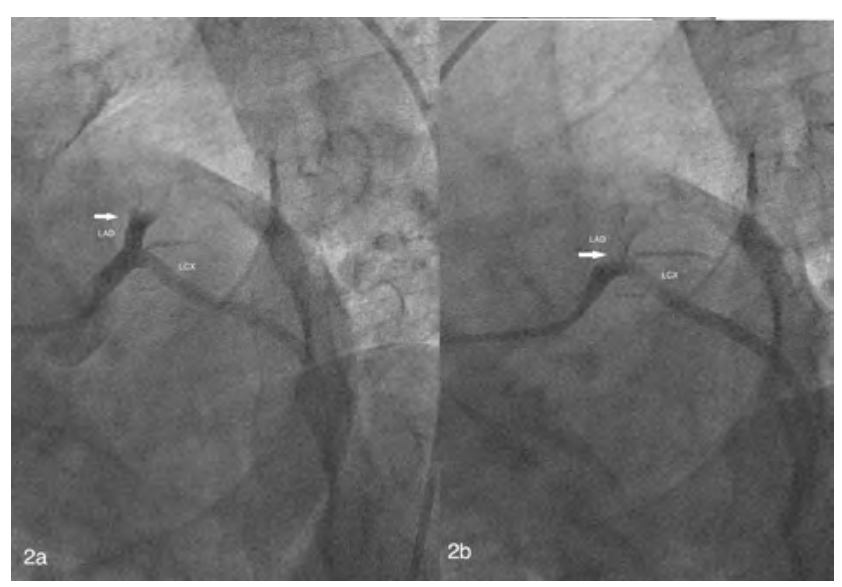

Figure 2

Figure 2a. Coronary angiography. Left anterior oblique caudal view: thrombus in the left anterior descending artery (LAD) and the first diagonal branch causing total occlusion (white arrow). Sluggish flow noted in the left circumflex artery (LCX) due to thrombus

Figure 2b. Coronary angiography. Left anterior oblique caudal view: thrombus in the ostial left anterior descending artery (LAD) causing total occlusion (white arrow). Sluggish flow noted in the left circumflex artery (LCX) due to thrombus

Five days later, patient was scheduled for elective $\mathrm{CAG}$ to reevaluate the lesion. $\mathrm{CAG}$ via right femoral artery approach was done which revealed normal left coronary artery with complete resolution of the thrombus (Figure 3). At biochemical analysis a moderately high serum homocysteine level (42 $\mu \mathrm{mol} / \mathrm{L}$; reference range: 5.46$16.20 \mu \mathrm{mol} / \mathrm{L})$ was found. Serum vitamin B12 was 258 $\mathrm{pg} / \mathrm{mL}$ (reference range: 211-911 pg/mL). Routine blood investigations including platelets, bleeding time, clotting time and activated partial thromboplastin time were normal, together with the normal renal and serum lipid profiles. Therefore, oral folic acid supplementation was initiated. 


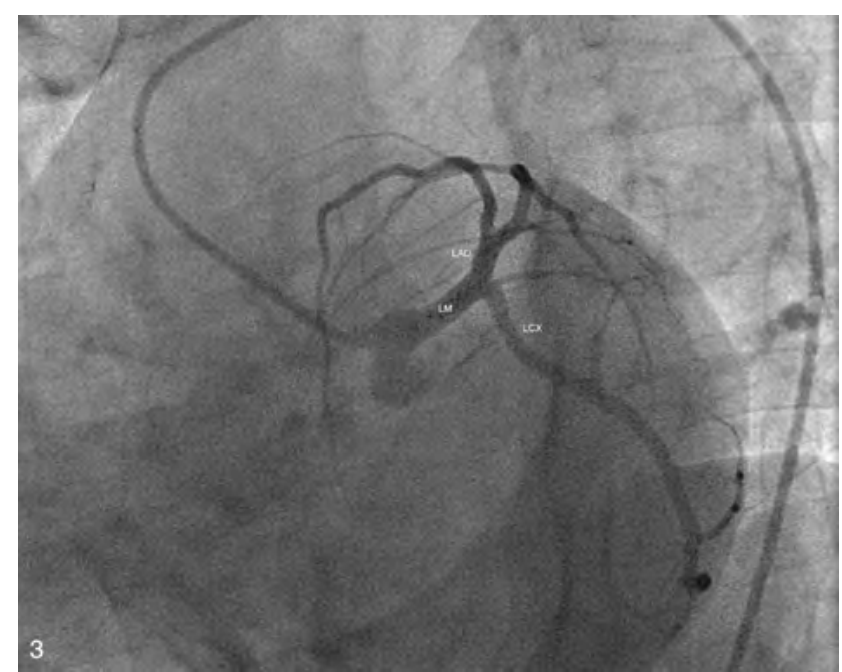

Figure 3

Figure 3. Repeat coronary angiography at five days. The intracoronary thrombus had disappeared and there was no residual coronary artery disease.

Patient's subsequent hospital stay was uneventful and discharged the next day free of angina. At one-year follow-up, patient remained symptom free but refused for examinations of repeat plasma homocysteine level as well as for other thrombophilia profile.

\section{DISCUSSION}

Coronary artery thrombosis commonly occurs in acute coronary syndrome secondary to plaque erosion or rupture of an atherosclerotic plaque. ${ }^{4}$ Sometimes, it may occur as a complication of invasive procedures. Other possible conditions like embolism, vasospasm, hypercoagulable states, antiphospholipid syndrome, nonatherosclerotic coronary diseases, trauma, hypoxia, intense sympathetic stimulation, and endothelial dysfunction can lead to thrombosis in normal coronary arteries..$^{5.6}$

We present a case of proximal LAD thrombus which got embolized to the ostial LAD during CAG leading to extensive anterior wall myocardial infarction which was successfully managed by intravenous thrombolysis. The exact cause of the coronary artery thrombosis in this patient is not known. Our patient had increased serum homocysteine level, thus, hyperhomocysteinemia could be the possible explanation for the development of coronary artery thrombosis in our patient. Hyperhomocysteinemia is a prothrombotic disorder and several studies have reported an increased plasma homocysteine level as a risk factor for occlusive arterial or venous disease. ${ }^{1,7}$ Many studies have shown that hyperhomocysteinemia was associated with an increased risk of coronary artery disease. ${ }^{8,9}$ It has been reported that moderate hyperhomocysteinemia are characterized by a prothrombotic, and dysfibrinolytic state and homocysteine level is an independent predictor of thrombotic events. ${ }^{2}$ The oxidative injury of endothelium in homocysteinemia, combined with the lack of vasculoprotective effects of nitric oxide, predisposes to thrombotic events. ${ }^{2}$ However, other thrombophilia profile could not be evaluated in our patient. Atherosclerotic disease is also kept in mind because our patient was a middle-aged smoker male, however, his ECG on presentation had no significant ST-T changes and also the follow-up CAG revealed no angiographic evidence of coronary artery disease. Procedure related catheter-induced coronary artery dissection with resultant thrombosis is known during coronary catheterization. But in our case, thrombus was seen in the first injection to the left coronary artery. Moreover, there was no catheter-induced dissection or vasospasm.

\section{CONCLUSION}

Hyperhomocysteinemia is associated to arterial thrombosis and is a risk factor of coronary artery disease. We presented a rare case of hyperhomocysteinemia and coronary artery thrombosis in the LAD artery causing total occlusion. The patient was successfully managed by intravenous thrombolysis. This case brings to light a rare case of hyperhomocysteinemia and coronary artery thrombosis.

\section{LEARNING POINTS}

- Hyperhomocysteinemia may lead to arterial thrombosis and should be in a list of differential diagnosis in any patient with coronary artery thrombosis.

- Serum homocysteine level should be measured in patients with coronary artery thrombosis to rule out hyperhomocysteinemia.

- There are two possible strategies in the management of coronary artery thrombosis. One is the interventional strategy (stenting preceded by thrombus aspiration) and another is the pharmacological strategy (GP IIb/ IIIa inhibitor or thrombolysis), eventually followed by an interventional strategy.

- This case brings to light a rare case of hyperhomocysteinemia and coronary artery thrombosis which was managed successfully with thrombolysis. 


\section{REFERENCES:}

1. Stampfer MJ, Malinow MR, Willett WC, et al. A prospective study of plasma homocyst(e)ine and risk of myocardial infarction in US physicians. JAMA 1992; 268:877-81. http://dx.doi.org/10.1001/ jama.268.7.877 http://dx.doi.org/10.1001/ jama.1992.03490070059042

2. Bienvenu T, Ankri A, Chadefaux B, et al. Elevated total plasma homocysteine, a risk factor for thrombosis. Relation to coagulation and fibrinolytic parameters. Thromb Res. 1993; 70:123-129. http://dx.doi.org/10.1016/00493848(93)90153-F

3. Christen WG, Ajani UA, Glynn RJ, et al. Blood levels of homocysteine and increased risks of cardiovascular disease: causal or casual? Arch Intern Med 2000; 160:422-34 http://dx.doi. org/10.1001/archinte.160.4.422

4. Farb A, Burke AP, Tang AL, et al. Coronary plaque erosion without rupture into a lipid core: a frequent cause of coronary thrombosis in sudden coronary death. Circulation 1996; 93:1354-63. http://dx.doi.org/10.1161/01.CIR.93.7.1354

5. Tun A, Khan IA. Myocardial infarction with normal coronary arteries: the pathologic and clinical perspectives. Angiology 2001; 52:299-304. http://dx.doi.org/10.1177/000331970105200501
6. Panduranga P, Riyami AA. Acute intracoronary thrombosis in a normal coronary artery following coronary angiography: thromboaspiration using a guide catheter. Heart Views 2010; 11:68-70. http:// dx.doi.org/10.4103/1995-705X.73218

7. den Heijer M, Blom HJ, Gerrits WB, et al. Is hyperhomocysteinaemia a risk factor for recurrent venous thrombosis? Lancet 1995; 345:882-5. http:// dx.doi.org/10.1016/S0140-6736(95)90008-X

8. Boushey CJ, Beresford SA, Omenn GS, et al. A quantitative assessment of plasma homocysteine as a risk factor for vascular disease: probable benefits of increasing folic acid intakes. JAMA 1995; 274:1049-57. http://dx.doi.org/10.1001/ jama.274.13.1049 http://dx.doi.org/10.1001/ jama.1995.03530130055028

9. Giles WH, Croft JB, Greenlund KJ, et al. Association between total homocyst(e)ine and the likelihood for a history of acute myocardial infarction by race and ethnicity: results from the Third National Health and Nutrition Examination Survey. Am Heart J 2000; 139:446-53. http:// dx.doi.org/10.1016/S0002-8703(00)90088-7 